\title{
UAV-Ground BS Coordinated NOMA with Joint User Scheduling, Power Allocation and Trajectory Design
}

\author{
Haiyong Zeng*, Xu Zhu*†, Yufei Jiang* and Zhongxiang Wei* \\ * School of Electronic and Information Engineering, Harbin Institute of Technology, Shenzhen, China \\ $\dagger$ School of Electrical Engineering and Electronics, University of Liverpool, Liverpool, UK \\ * School of Electrical Engineering and Electronics, University College London, London, UK \\ Emails: zenghaiyong@stu.hit.edu.cn, xuzhu@liverpool.ac.uk, jiangyufei@hit.edu.cn, zhongxiang.wei@ucl.ac.uk
}

\begin{abstract}
We propose an unmanned aerial vehicle (UAV)ground base station (GBS) coordinated NOMA scheme where UAV and GBS are jointly serve the cell-edge users. To the best of our knowledge, this is the first work to investigate airground BSs coordination for UAV-assisted NOMA systems, by taking advantage of the interference between UAV and GBS. Therefore, the proposed UAV-GBS coordinated NOMA scheme achieves much higher sum rate of cell-edge users than the noncoordinated UAV-assisted NOMA schemes where interference is suppressed as much as possible. The proposed scheme also leads to better channel condition and more flexible and cost-effective interference management, compared to GBSs coordinated NOMA. We conduct joint optimization of power allocation, user scheduling and UAV trajectory for the UAV-GBS coordinated system. A closed-form optimal solution to power allocation is derived. In addition, a dedicated successive interference cancellation (SIC) ordering approach is proposed. It is proven that the selection of a cell-center user with higher SIC order contributes to a higher rate of cell-edge user, based on which an SIC order based user scheduling algorithm for both cell-center and cell-edge users is presented.
\end{abstract}

\section{INTRODUCTION}

As one of the promising techniques for fifth generation $(5 \mathrm{G})$ and beyond $5 \mathrm{G}$ wireless communication, non-orthogonal multiple access (NOMA) provides considerable spectrum efficiency enhancement over conventional orthogonal multiple access (OMA) techniques [1]. By utilizing superposition coding at the transmitting side for power-domain multiplexing, and successive interference cancellation (SIC) at the receiver for signal detection, power-domain NOMA allows multiple users to be served with the same frequency, time and code resource element [2].

Though NOMA can achieve enhanced spectrum efficiency, the quality of service (QoS) of cell-edge users is often a bottleneck of the network performance especially in a special event with a large number of users [3]-[5]. As mentioned in [6]-[8], owing to flexibility and a better air-ground channel with line of sight (LoS) link, unmanned aerial vehicles (UAVs) have drawn increasing attention and are being widely deployed to serve as temporary mobile base stations (BSs). A hybrid scenario was proposed in [7] where the UAV-BS cooperates with ground BS (GBS) to provide access services to offload traffic of GBS. In [8], UAV-BS was employed at the edge of multiple adjacent cells to help improve the performance of celledge users and offload traffic of GBSs.
In UAV-assisted NOMA systems, due to the presence of GBS, the performance of users can be severely affected by the interference between UAV and GBS [9] [10] et al.. In [9], Nguyen proposed a cooperative UAV-NOMA scheme in wireless backhaul networks, and the UAV trajectory, SIC order and NOMA beamforming vectors were jointly optimized to maximize the sum rate of UAV-served users. However, the interference of GBS to UAV-served users was not taken into account. In [10], a NOMA precoding matrix was proposed for the multi-antenna GBS so that the interference from GBS to UAV-served users can be zero-forced or restricted to a given threshold, and the UAV trajectory is optimized to maximize the sum rate of cell-edge users. However, their design of NOMA precoding matrix is complex, and it requires additional multiple antennas to perform the precoding matrix. In [11], coordination between GBSs (referred to as coordinated multi-point with joint transmission) was proposed to allow joint signal transmission for the cell-edge users in a NOMA system. It was proven in [11] that the NOMA with coordinated BSs can take advantage of the space diversity and provide higher performance than non-coordinated NOMA systems. Hence, it is preferable to investigate coordination of UAV-BS and GBS in a UAV-assisted NOMA system to utilize interference rather than suppress it as much as possible. To the best of our knowledge, this work has not been reported in the literature.

Resource allocation plays a crucial role in a UAV assisted NOMA system, which includes user scheduling, power allocation and UAV trajectory design. Due to deployment of UAV, the previous work on resource allocation for GBSs coordinated NOMA [11] is not applicable. In addition, the user scheduling and UAV trajectory optimization algorithms dedicated for UAVGBS coordinated OMA [12] or non-coordinated UAV-assisted NOMA system [10] are not suitable for UAV-GBS coordinated NOMA systems. The resource allocation for UAV-GBS coordinated NOMA still remains an open challenge in the literature.

Motivated by the above open issues, we propose a UAVGBS coordinated NOMA scheme which allows UAV and GBS to serve the cell-edge users simultaneously via joint signal transmission. Effective user scheduling and power allocation alongside UAV trajectory design are investigated to maximize the sum rate of cell-edge users. The contributions of this paper are summarized as follows:

1) To the best of our knowledge, this is the first work 
to investigate air-ground BSs coordination for UAV-assisted NOMA systems, by taking advantage of the interference between UAV and GBS rather than suppressing it as much as possible like in [9] and [10]. Therefore, the proposed UAVGBS coordinated NOMA scheme achieves much higher sum rate of cell-edge users than the non-coordinated UAV-assisted NOMA schemes in [9] and [10]. Also, the proposed UAV-GBS coordinated NOMA scheme leads to better channel condition with air-ground LoS link and more flexible and cost-effective interference management, thanks to UAV's mobility, compared to GBSs coordinated NOMA [11].

2) We make the first study of joint optimization of power allocation, user scheduling, UAV trajectory for the proposed UAV-GBS coordinated NOMA system. Since UAV is mobile and multiple users can share the same frequency and time in NOMA, the previous work on resource allocation for UAVGBS coordinated OMA [12] or GBSs coordinated NOMA [11] or non-coordinated UAV-assisted NOMA [9] [10] is not applicable. An efficient iterative algorithm is proposed to solve the optimization problem, assisted by a novel SIC ordering approach that is based on initial channel-to-interference-andnoise (I-CINR) ratio for cell-center users.

3) An intensive theoretical analysis is presented. A closedform optimal solution to power allocation is derived by converting a non-convex optimization problem into convex without any loss of optimality, while no closed-form solutions to power allocation were provided in the previous work on UAV-assisted NOMA [9] [10]. In addition, we conduct user scheduling for both cell-edge and cell-center users, based on the SIC ordering approach proposed, while scheduling of cell-edge users only was considered in [10]. The users with higher SIC decoding order are scheduled first, as it is proven that those users contribute to a higher achievable rate of cell-edge user.

The rest of this paper is organized as follows. The system model and optimization problem are formulated in Section II. The closed-form optimal power allocation is given in Section III. The user scheduling algorithm is proposed in Section IV. Section V designs the UAV trajectory. Numerical results are presented in Section VI. Section VII concludes this paper.

\section{System Model ANd PRoblem Formulation}

\section{A. System Model}

We consider a downlink UAV-assisted NOMA system with a UAV-BS, a GBS and $K$ ground users, both the BSs and users are equipped with a single antenna. The UAV and GBS are connected to a central unit through high-capacity links [12]. As depicted in Fig. 1, the $K$ ground users are classified into $K_{c}$ cell-center users and $K_{e}$ cell-edge users based on a threshold of distance to GBS [8]. The cell-center users are served by GBS based on NOMA strategy while the cell-edge users are associated to UAV. Note that the UAV-served users can suffer from severe interference from GBS, to alleviate this and improve the performance of cell-edge users, they are also served by GBS. Denote the sets of cell-center and cell-edge users as $k \in \mathbf{K}_{\mathbf{c}}=\left\{1,2, \ldots, K_{c}\right\}$ and $l \in \mathbf{K}_{\mathbf{e}}=\left\{1,2, \ldots, K_{e}\right\}$, respectively.

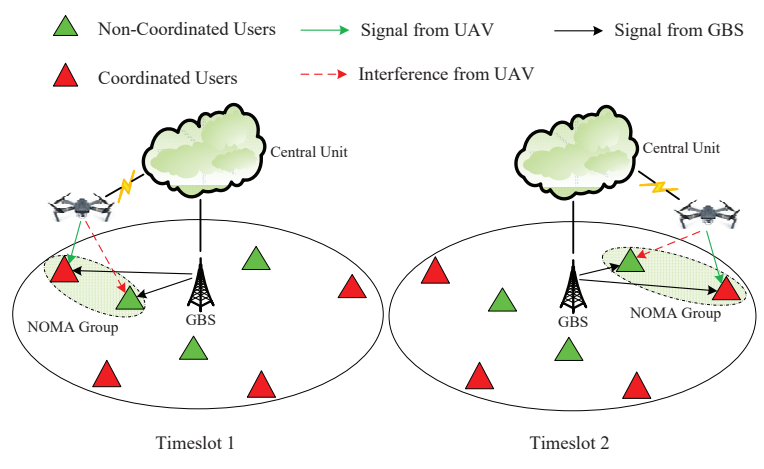

Fig. 1. System model of UAV-GBS coordinated NOMA.

Assume the UAV flies periodically above the cell with a fixed height $H$ and a constant cycle flight time $T$, which can be equally discretized into $N$ time slots. Considering the horizontal coordinates, the location of UAV projected on the horizontal ground at time slot $n$ can be denoted as $\mathbf{q}_{n}=[x[n], y[n]]^{T}$. Also, we denote the positions of GBS and an arbitrary user $i$ as $\mathbf{L}_{b}=\left[x_{b}, y_{b}\right]^{T}$ and $\mathbf{L}_{i}=\left[x_{i}, y_{i}\right]^{T}$, respectively. As mentioned in [9], the air-to-ground (AtG) communication from UAV to ground users is governed by LoS propagation. Assume the Doppler effect caused by the UAV's mobility can be successfully compensated [7], the AtG channel from UAV to an arbitrary ground user $i$ at time slot $n$ is $v_{i, n}=\sqrt{\frac{\beta_{u}}{H^{2}+\left\|\mathbf{q}[n]-\mathbf{L}_{i}\right\|^{2}}}$, in which $\beta_{u}$ stands for the channel power gain at the reference distance $d_{0}=1 \mathrm{~m}$ from the UAV [8]. On the other hand, the channel frequency response from GBS to user $i$ at time slot $n$ can be obtained as $h_{b, i, n}=g_{b, i, n} P L\left(d_{i}\right)$, with $g_{b, i, n}$ as the Rayleigh fading channel gain, and $P L\left(d_{i}\right)$ as the path loss function [13].

According to NOMA strategy, multiple users can share the same frequency resource at each time slot. Define $C_{i, n} \in$ $\{0,1\}$, where $C_{i, n}=1$ indicates that user $i$ is scheduled at time slot $n$. Following [10] and [13], to keep low SIC decoding complexity at the receiver and restrict the error propagation, we consider there are $G$ users share the same frequency at each time slot including $(G-1)$ cell-center users and one cell-edge user. Denote the set of users scheduled at time slot $n$ as $U_{n}$. Then the received signals at user $i$ can be written as

$$
y_{i, n}=\sum_{j \in U_{n}} h_{i, n} \sqrt{p_{j, n}} \theta_{j, n}+v_{i, n} \sqrt{p_{u}} \theta_{l, n}+z_{i, n},
$$

where $\theta_{j, n}$ and $\theta_{l, n}$ denote the transmitted symbols with unit energy, $p_{u}$ is the transmit power of UAV and $p_{i, n}$ denotes the transmit power of user $i$ from GBS at time slot $n$, respectively. $z_{i, n}$ is the additive white Gaussian noise $z_{i, n} \sim C N\left(0, \sigma^{2}\right)$.

At the receiver, SIC is conducted to decode the received signals. Define $H_{i, n}=\left|h_{i, n}\right|^{2}$ and $V_{i, n}=\left|v_{i, n}\right|^{2}$ as the channel gain of user $i$ from GBS and from UAV, respectively. The multiplexed users can be decoded based on an SIC order based on channel gain [13] or the proposed SIC decoding order in Section III. For a cell-center user $k$, the achievable data rate 
(in bps/Hz) after SIC can be obtained as

$$
R_{k, n}=\log _{2}\left(1+\frac{H_{k, n} P_{k, n}}{\sum_{j \in U_{k, n}} H_{k, n} P_{j, n}+V_{k, n} P_{u}+\sigma^{2}}\right) .
$$

where $U_{k, n}$ denotes the set of users in $U_{n}$ with a higher SIC decoding order than user $k$. On the other hand, for a celledge user $l$, since it is jointly served by UAV and GBS, the achievable data rate of user $l$ after SIC is given by

$$
R_{l, n}=\log _{2}\left(1+\frac{H_{l, n} P_{l, n}+V_{l, n} P_{u}}{\sigma^{2}+\sum_{k \in U_{l, n}} H_{l, n} P_{k, n}}\right) .
$$

in which the term $\sum_{k \in U_{l, n}} H_{l, n} P_{k, n}$ denotes the SIC interference from the set of users with a higher SIC order than user $l$ in $U_{n}$. The mean sum rate of cell-edge users can be obtained as

$$
R_{\text {sum }}^{e}=\frac{1}{N} \sum_{n=1}^{N} \sum_{l \in K_{e}} C_{l, n} R_{l, n}
$$

\section{B. Problem Formulation}

In this subsection, we dedicate to maximizing the sum rate of cell-edge users by jointly optimizing the user scheduling matrix $\mathbf{C}$, power allocation matrix $\mathbf{P}$ and UAV trajectory matrix $\mathbf{Q}$, with a minimum rate constraint for the scheduled cell-center users in each time slot. The optimization problem for the UAVGBS coordinated NOMA can be formulated as

$$
\begin{aligned}
& \text { OP1 : } \max _{\mathbf{C}, \mathbf{P}, \mathbf{Q}} R_{\text {sum }}^{e} \\
& \text { s.t.(C1) } P_{j, n} \geq 0, \forall j \in U_{n} ; \\
& \quad(C 2) \sum_{j \in U_{n}} P_{j, n} \leq P_{t}, \forall n \in \mathbf{N} ; \\
& \quad(C 3) R_{k, n} \geq R_{\min }, \forall k \in\left\{U_{n} \cap K_{c}\right\}, n \in \mathbf{N} ; \\
& \quad(C 4) C_{i, n} \in\{0,1\}, \forall i \in \mathbf{K} ; \\
& \quad(C 5) \sum_{k \in K_{c}} C_{k, n}=G-1, \forall n \in \mathbf{N} ; \\
& \quad(C 6) \sum_{l \in K_{e}} C_{l, n}=1, \forall n \in \mathbf{N} ; \\
& \text { (C7) } \sum_{n=1}^{N} C_{k, n} \leq \alpha, \forall k \in K_{c} ;(C 8) \sum_{n=1}^{N} C_{l, n} \leq \beta, \forall l \in K_{e} ; \\
& \quad(C 9) \mathbf{q}[1]=\mathbf{q}[N] ; \\
& (C 10)\|\mathbf{q}[n+1]-\mathbf{q}[n]\|^{2} \leq\left(v_{\max } T / N\right)^{2}, \forall n \in \mathbf{N},
\end{aligned}
$$

where $(C 1)$ and $(C 2)$ are the transmission power budget. (C3) ensures the QoS of the scheduled cell-center users. $(C 4)-(C 6)$ denote the user scheduling constraints. In addition, considering user fairness, $(C 7)$ and $(C 8)$ are presented to constraint the maximum number of time slots occupied by each users. Note that the values of $\alpha$ and $\beta$ can be adaptively adjusted according to the QoS requirement of users. Furthermore, $(C 9)$ assures a periodic flight and $(C 10)$ constraints the maximum speed of UAV $v_{\max }$. The energy efficiency (EE) of the UAV-GBS coordinated NOMA system is given by $E E=\frac{B}{N} \sum_{n=1}^{N} \frac{\sum_{k=1}^{K_{c}} C_{k, n} R_{k, n}+\sum_{l=1}^{K_{e}} C_{k, n} R_{l, n}}{P\left(v_{n}\right)+P_{u}+\sum_{j \in U_{n}} P_{j, n}}$, with $B$ denoting the total bandwidth and $P\left(v_{n}\right)$ as the propulsion power of UAV which can be taken as a function of flying speed $v_{n}$ [4].

It is obvious that the optimization problem in OP1 under constraints $(C 1)-(C 10)$ is constrained combinatorial nonconvex, and it requires considerable complexity to obtain the global optimal solution. To address this problem efficiently, OP1 is decoupled into three sub-problems, namely user scheduling, power allocation and UAV trajectory, and a suboptimal low complexity solution can be achieved by alternately solving the sub-problems [8] [10].

\section{SIC ORDERING AND Optimal POWER Allocation FOR UAV-GBS COORDINATED NOMA}

\section{A. SIC Ordering}

As mentioned in [13], for single-cell NOMA systems, the optimal decoding order in SIC process is based on the increasing order of channel gains from serving BS. Nevertheless, in UAVGBS coordinated NOMA systems, due to the effect of UAV, the user with a higher channel gain from GBS cannot guarantee a better the signal-to-interference-plus-noise ratio (SINR) than other users, which makes the SIC order based on channel gain inefficient. As mentioned above, there are $G$ users in NOMA group $U_{n}$. Denote the indexes of cell-center users as $1, \ldots, G-1$ and the cell-edge user as $G$, respectively. Without loss of generality, we assume the SIC decoding order of users in $U_{n}$ is $1,2, \ldots, G-1, G$. Since the cell-edge user generally suffers from poor channel condition due to the long distance from GBS, it can be taken as weak user in comparison to the cell-center users. Denote the I-CINR of user $k$ as $\Phi_{k, n}=\frac{H_{k, n}}{\sigma^{2}+V_{k, n} P_{u}}$.

Lemma 1: In UAV-GBS coordinated NOMA systems, given an SIC order of $1, \ldots, G$, in order to successfully perform SIC, the users in NOMA group $U_{n}$ should satisfy

$$
\Phi_{1, n} \geq \ldots \geq \Phi_{k, n} \geq \ldots \geq \Phi_{G-1, n} \geq \frac{H_{G, n}}{\sigma^{2}} .
$$

Proof of Lemma 1: First, for a center user $k$ in $U_{n}$, the SINR of user $k+1$ at user $k$ takes the expression as

$$
\gamma_{k \rightarrow k+1, n}=\frac{H_{k, n} P_{k+1, n}}{\sigma^{2}+\sum_{j=1}^{k} H_{k, n} P_{j, n}+V_{k, n} P_{u}} .
$$

To successfully decode the signal of user $k+1$ at user $k$, we should have $\gamma_{k \rightarrow k+1, n} \geq \gamma_{k+1, n}$, according to (2) and (6), we have $\Phi_{k, n} \geq \Phi_{k+1, n}, k=1, \ldots, G-2$.

For the cell-edge user $G$, the SINR of user $G$ at user $k$ is

$$
\gamma_{k \rightarrow G, n}=\frac{H_{k, n} P_{G, n}}{\sigma^{2}+\sum_{j=1}^{k} H_{k, n} P_{j, n}+V_{k, n} P_{u}}
$$

Since the cell-edge user $G$ is jointly served by GBS and UAV, to successfully decode the signal from GBS to user $G$ at user $k$, we have

$$
\gamma_{k \rightarrow G, n} \geq \frac{H_{G, n} P_{G, n}}{\sigma^{2}+\sum_{k=1}^{G-1} H_{G, n} P_{k, n}}
$$


which implies $\Phi_{k, n} \geq H_{G, n} / \sigma^{2}, k=1, \ldots, G-1$.

\section{B. Closed-Form Optimal Solution to Power Allocation}

Given UAV trajectory $\mathbf{Q}$ and user scheduling $\mathbf{C}$, the power allocation problem can be formulated as

$$
\begin{aligned}
& \text { OP2 } \max _{\mathbf{P}} R_{\text {sum }}^{e} \\
& \text { s.t. }(C 2),(C 3) \text { and }(C 4) .
\end{aligned}
$$

OP2 is non-convex in terms of $\mathbf{P}$ and hard to solve. Hence, Theorem 1 is proposed to transform OP2 into a convex problem without any loss of optimality.

Theorem 1: In UAV-GBS coordinated NOMA, the optimal solution to the power allocation problem OP2 can be found by equivalently solving the convex optimization problem as

$$
\begin{aligned}
& \text { OP3 : } \max _{\mathbf{R}_{n}} R_{\text {sum }}^{e} \\
& \text { s.t. }(\widetilde{C 3}) P_{n}\left(\mathbf{R}_{\mathbf{n}}\right) \leq P_{t}, \forall n \in \mathbf{N} ;(C 4) ; \\
& (C 11) R_{i, n} \geq 0, \forall i \in U_{n} ;
\end{aligned}
$$

where $P_{n}\left(\mathbf{R}_{\mathbf{n}}\right)=2^{R_{G, n}}\left(\frac{\sigma^{2}}{H_{G, n}}-\frac{1}{\Phi_{G-1, n}}\right)-\frac{1}{\Phi_{G, n}}+$

$$
\sum_{k=2}^{G-1}\left(\frac{1}{\Phi_{k, n}}-\frac{1}{\Phi_{k-1, n}}\right) 2^{\sum^{G} R_{k+1}}+2^{\sum_{j=1}^{G} R_{j, n}} \frac{1}{\Phi_{1, n}}
$$

Proof of Theorem 1: See Appendix A.

Based on Thereom 1, differentiating $\sum_{k=1}^{G-1} P_{k, n}$ with respect to the rate of cell-center user $k R_{k, n}$ yields

$\frac{\partial \sum_{k=1}^{G-1} P_{k, n}}{\partial R_{k, n}}=\frac{2^{\sum_{k=1}^{G-1} R_{k, n}} \ln 2}{\Phi_{1, n}}+2^{\sum_{j=k}^{G-1} R_{k, n}} \ln 2 \sum_{k=2}^{G-1}\left(\frac{1}{\Phi_{k, n}}-\frac{1}{\Phi_{k-1, n}}\right)$

According to Lemma 1, we have $\frac{\partial \sum_{k=1}^{G-1} P_{k, n}}{\partial R_{k, n}} \geq 0$, which indicates that $\sum_{k=1}^{G-1} P_{k, n}$ is monotonically increasing with respect to $R_{k, n}$. Moreover, it can be learned from (3) that a smaller $\sum_{k=1}^{G-1} P_{k, n}$ leads to less SIC interference and a higher available transmission power for cell-edge user $G$. As a consequence, to minimize the sum power of cell-center users and maximize the performance of cell-edge users, the rates of cell-center user $k$ in time slot $n$ should be $R_{k, n}^{*}=R_{\text {min }}$. Substituting $R_{k, n}^{*}$ into (3), we obtain the optimal sum rate of cell-edge user $G$ for time slot $n$ as

$$
\begin{aligned}
& R_{G, n}^{*}=\log _{2}\left(1+\left(H_{G, n} P_{G, n}+V_{G, n} P_{u}\right) /\right. \\
& \left(\sigma^{2}+H_{G, n} \times\left(\frac{\sigma^{2}+V_{1, n} P_{u}}{H_{1, n}} 2^{\sum_{k=1}^{G-1} R_{k, n}^{*}}-\frac{\sigma^{2}+V_{G-1, n} P_{u}}{H_{G-1, n}}\right.\right. \\
& \left.\left.\left.+\sum_{k=2}^{G-1}\left(\frac{\sigma^{2}+V_{k, n} P_{u}}{H_{k, n}}-\frac{\sigma^{2}+V_{k-1, n} P_{u}}{H_{k-1, n}}\right) 2^{\sum_{j=k}^{G-1} R_{k, n}^{*}}\right)\right)\right) .
\end{aligned}
$$

\section{UsER Scheduling For UAV-GBS COORDinAted NOMA}

In this section, we propose an SO-US algorithm where the cell-center users are scheduled by their corresponding ICINRs and the cell-edge users are selected according to the approximated SINR.

According to Lemma 1, the SIC order of cell-center users is the increasing order of I-CINR.

Lemma 2: In UAV-GBS coordinated NOMA systems, the selection of a cell-center user with larger $\Phi$ contributes to a higher sum rate of cell-edge users than any other cell-center users with lower $\Phi$.

Proof of Lemma 2: According to Thereom 1, the transmit power of an arbitrary cell-center user $k$ can be obtained as

$$
P_{k, n}=\left(2^{R_{\min }}-1\right)\left(\frac{1}{\Phi_{k, n}}+\sum_{j=1}^{k-1} P_{j, n}\right)
$$

where $\sum_{j=1}^{k-1} P_{j, n}$ is the sum power of the first $k-1$ scheduled users, which is predetermined. Given a larger $\Phi_{k, n}$, the transmit power of user $k$ will be smaller. According to (3) and constraint $(C 3)$, the SIC interference from user $k$ to user $G$ decreases and there is more available transmit power in GBS to serve the cell-edge user $G$, which leads to a higher $R_{G, n}$.

Moreover, notice that user scheduling, power allocation and trajectory design can be solved alternatively in each iteration to convergence to a best one [3]. Assume that the transmit power of cell-edge user $l$ from GBS in the last iteration is $P_{l, n}^{i-1}$, the approximated SINR of user $l$ at iteration $i$ can be obtained as

$$
\gamma_{l, n}^{i}=\frac{H_{l, n} P_{l, n}^{i-1}+V_{l, n} P_{u}}{\sigma^{2}}+H_{l, n}\left(P_{i-1}-P_{l, n}^{i}\right) .
$$

Compared to the previous user scheduling schemes based on channel gain [14], the proposed SO-US algorithm takes a comprehensive consideration of users' channel gain and interference, which is more efficient. In addition, by directly feeding the user ranking list to SIC decoding process, the proposed SO-US has lower SIC complexity over the previous work based on channel gain that requires an additional user ranking process in SIC decoding [14].

1) Scheduling for Cell-Center Users: For each time slot $n, n=1, \ldots, N$, the $K_{c}$ cell-center users are ranked in descending order based on their I-CINRs, and forms a $1 \times K_{c}$ ranking list $\Gamma_{n}$. Then, from $n=1$ to $N$, while the number of users in time slot $n$ is less than $G-1$, for each user $k$ starting from the top of list $\Gamma_{n}$, check the number of time slots assigned to user $k$, if $\sum_{i=1}^{n-1} C_{k, i}<\alpha$, user $k$ is directly assigned to time slot $n$ and set $C_{k, n}=1$; otherwise, according to the time sequence property, user $k$ is removed from $\Gamma_{n}$. Repeat the procedures until the $K_{c}$ cell-center users and $N$ time slots are scheduled. The user scheduling for cell-center users is described in Algorithm 1.

2) Scheduling for Cell-Edge Users: For the scheduling of cell-edge users, from $n=1$ to $N$, calculate the approximated SINR of each cell-edge user $j\left(j=1, \ldots, K_{e}\right)$ by $(10)$. After 


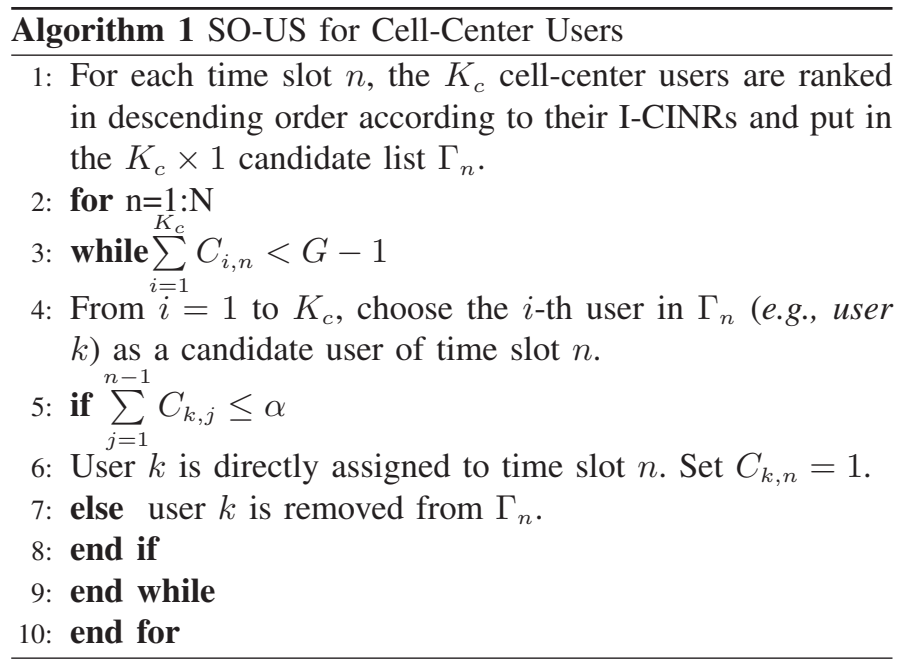

that, the $K_{e}$ cell-edge users are ranked in descending order based on their approximated SINRs $\gamma_{l, n}^{i}$ in each time slot $n$, and forms a $1 \times K_{e}$ candidate list $\eta_{n}$. Then, from $n=1$ to $N$, while $\sum_{j=1}^{K_{e}} C_{j, n}<1$, the top user in $\eta_{n}$ (e.g., user $l$ ) is selected as the candidate user for time slot $n$. Similar to Algorithm 1, if $\sum_{j=1}^{n-1} C_{l, j}<\beta$, user $l$ is assigned to time slot $n$ and set $C_{l, n}=1$. Otherwise, remove user $l$ from $\eta_{n}$. Repeat the steps above until the $K_{e}$ users and $N$ time slots are assigned.

\section{TRAJECTORY Design}

In this section, based on the user scheduling $\mathbf{C}$ and power allocation $\mathbf{P}$ presented in Sections III and IV, respectively, we attempt to design the UAV trajectory to maximize the sum rate of cell-edge users. The UAV trajectory optimization subproblem for UAV-GBS coordinated NOMA system is

$\mathbf{O P 4}: \max _{\mathbf{Q}} \sum_{n=1}^{N} \sum_{l \in K_{e}} \log _{2}\left(1+\frac{H_{l, n} P_{l, n}+\frac{\beta_{u} P_{u}}{H^{2}+\left\|\mathbf{q}[n]-\mathbf{L}_{l}\right\|^{2}}}{\sigma^{2}+\sum_{k=1}^{G-1} H_{l, n} P_{k, n}}\right)$ s.t. $(C 9),(C 10)$, and

$(\widetilde{C 4}) \log _{2}\left(1+\frac{H_{k, n} P_{k, n}}{\sigma^{2}+\sum_{j=1}^{k-1} H_{k, n} P_{j, n}+\frac{\beta_{u} P_{u}}{H^{2}+\left\|\mathbf{q}[n]-\mathbf{L}_{k}\right\|^{2}}}\right) \geq R_{\min }$, which is non-convex. Following [8], OP4 can be approximated as a standard convex form by employing successive convex optimization methodology.

Note that constraint $(\widetilde{C 4})$ can be rewritten as

$$
\begin{aligned}
& H^{2}+\left\|\mathbf{q}[n]-\mathbf{L}_{k}\right\|^{2} \geq \\
& \left(2^{R_{\min }}-1\right) \frac{\beta_{u} P_{u}}{H_{k, n} P_{k, n}}-\left(\sigma^{2}+\sum_{j=1}^{k-1} H_{k, n} P_{j, n}\right),
\end{aligned}
$$

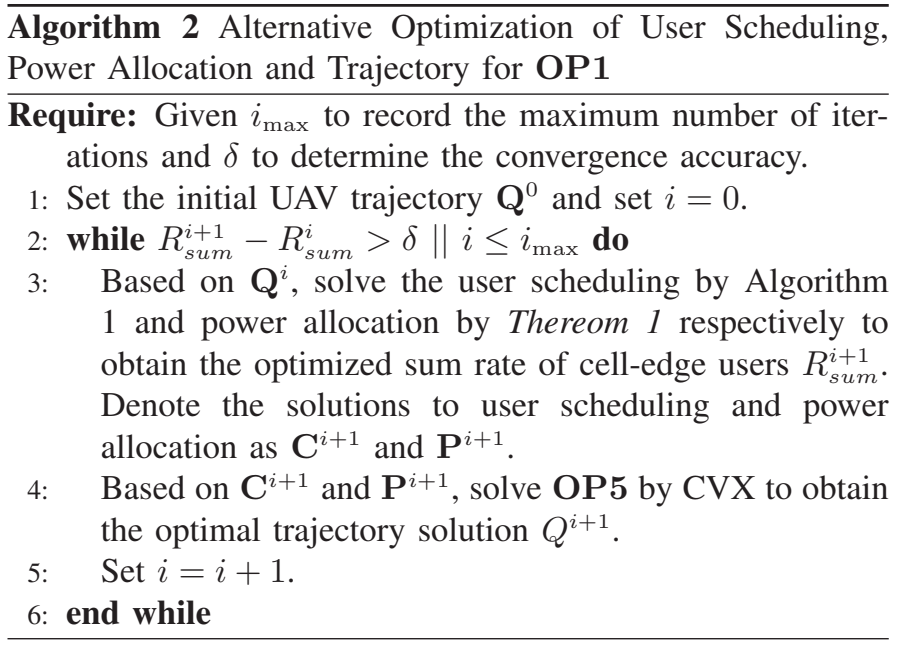

where $H^{2}+\left\|\mathbf{q}[n]-\mathbf{L}_{k}\right\|^{2}$ is convex with respect to $\left\|\mathbf{q}[n]-\mathbf{L}_{k}\right\|^{2}$. The lower bound of $H^{2}+\left\|\mathbf{q}[n]-\mathbf{L}_{k}\right\|^{2}$ at local point $\mathbf{q}^{i}[n]$ can be obtained as

$$
\begin{aligned}
H^{2}+\left\|\mathbf{q}[n]-\mathbf{L}_{k}\right\|^{2} & \geq H^{2}+\left\|\mathbf{q}^{i}[n]-\mathbf{L}_{k}\right\|^{2}+ \\
& 2\left(\mathbf{q}^{i}[n]-\mathbf{L}_{k}\right)^{T}\left(\mathbf{q}[n]-\mathbf{q}^{i}[n]\right) .
\end{aligned}
$$

Substituting (12) into (11) yields

$$
\begin{gathered}
\left(\widetilde{C{ }^{\prime}}\right) H^{2}+\left\|\mathbf{q}^{i}[n]-\mathbf{L}_{k}\right\|^{2}+2\left(\mathbf{q}^{i}[n]-\mathbf{L}_{k}\right)^{T}\left(\mathbf{q}[n]-\mathbf{q}^{i}[n]\right) \geq \\
\left(2^{R_{\min }}-1\right) \frac{\beta_{u} P_{u}}{H_{k, n} P_{k, n}}-\left(\sigma^{2}+\sum_{j=1}^{k-1} H_{k, n} P_{j, n}\right),
\end{gathered}
$$

which is now convex with respect to $\mathbf{q}[n]$.

Similarly, though $R_{l, n}$ is not convex with respect to $\mathbf{q}[n]$, it is a concave function of $\left\|\mathbf{q}^{i}[n]-\mathbf{L}_{l}\right\|^{2}$. As a result, the lower bound of $R_{l, n}$ during the $i$-th iteration $R_{l, n}^{i}$ can be obtained as

$$
R_{l, n}^{i} \triangleq \psi_{l}^{i}[n]\left(\left\|\mathbf{q}[n]-\mathbf{L}_{l}\right\|^{2}-\left\|\mathbf{q}^{i}[n]-\mathbf{L}_{l}\right\|^{2}\right)+\phi_{l}^{i}[n],
$$

where $R_{l, n}^{i}$ is the lower bound to $R_{l, n}$ at local point $\mathbf{q}^{i}$, and

$$
\begin{gathered}
\psi_{l}^{i}[n]=-\frac{\frac{\beta_{u} P_{u}}{\left(\sigma^{2}+\sum_{k=1}^{G-1} H_{l, n} P_{k, n}\right)\left(H^{2}+\left\|\mathbf{q}^{i}[n]-\mathbf{L}_{l}\right\|^{2}\right)^{2}} \log _{2}(e)}{1+\frac{H_{l, n} P_{l, n}+\frac{\beta_{u} P_{u}}{H^{2}+\left\|\mathbf{q}^{i}[n]-\mathbf{L}_{l}\right\|^{2}}}{\sigma^{2}+\sum_{k=1}^{G-1} H_{l, n} P_{k, n}}}, \\
\phi_{l}^{i}[n]=\log _{2}\left(\begin{array}{c}
1+\frac{H_{l, n} P_{l, n}+\frac{\beta_{u} P_{u}}{H^{2}+\left\|\mathbf{q}^{i}[n]-\mathbf{L}_{l}\right\|^{2}}}{\sigma^{2}+\sum_{k=1}^{G-1} H_{l, n} P_{k, n}}
\end{array}\right) .
\end{gathered}
$$

It can be learned from (13) that the lower bound $\tilde{R}_{l, n}^{i}$ is concave in terms of $\mathbf{q}[n]$. As a result, $\mathbf{O P} 4$ can be approximated as a convex problem with respect to $\mathbf{Q}$ as

$$
\begin{aligned}
& \text { OP5 } \max _{\mathbf{Q}} \sum_{n=1}^{N} \sum_{l \in K_{e}} \tilde{R}_{l, n}^{i} \\
& \text { s.t. }\left(\widetilde{C 4^{\prime}}\right),(C 9) \text { and }(C 10),
\end{aligned}
$$

which can be effectively solved by CVX. 


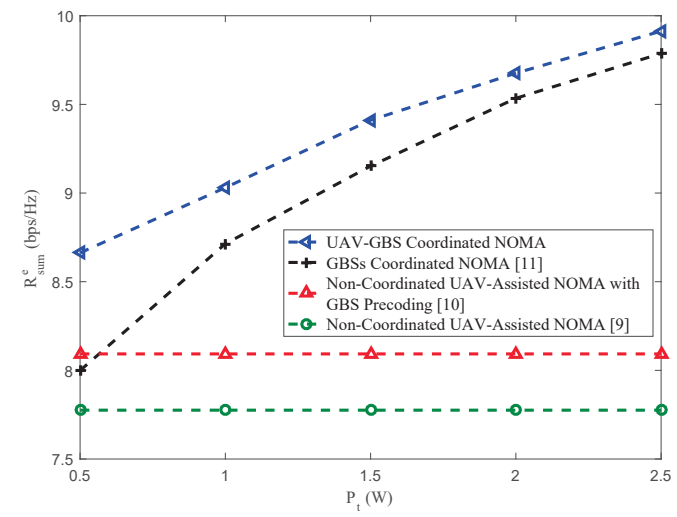

Fig. 2. Impact of the GBS transmit power $P_{t}$ on performance of various UAV-assisted NOMA and coordinated NOMA schemes.

By alternately solving user selection and power allocation by SO-US algorithm and Thereom 1, and UAV trajectory in OP 5 by CVX, respectively, the original problem OP1 can be solved. The alternative optimization algorithm for UAV-GBS coordinated NOMA is present in Algorithm 2.

\section{NumericAl RESUlts}

In this section, numerical results are presented to evaluate the performance of our proposed UAV-GBS coordinated NOMA scheme. Referring to [15], we set $N=60, H=50 \mathrm{~m}, T=100$ $\mathrm{s}$ and $v_{\max }=40 \mathrm{~m} / \mathrm{s}$. Following the topology in Fig. 1, we set $K_{c}=3, K_{e}=4$, and $G=2$, the bandwidth is set as $B=1$ $\mathrm{MHz}$. The path loss model from GBS to users is given as $P L(d)=128.1+37.6 \log 10(d)[13]$.

Fig. 2 shows the impact of the GBS transmit power $P_{t}$ on performance of various UAV-assisted NOMA and coordinated NOMA schemes, versus the transmit power of GBS. $P_{u}=20$ $\mathrm{mW}$ and $R_{\text {min }}=4 \mathrm{bps} / \mathrm{Hz}$. As can be seen, the performance of the proposed UAV-GBS coordinated NOMA scheme is substantially better than that of non-coordinated UAV-NOMA schemes [9] [10], which remain unchanged with the increasing of $P_{t}$. For example, when $P_{t}=1.5 \mathrm{~W}$, the proposed UAVGBS coordinated NOMA has about $21.03 \%$ and $16.29 \%$ higher $R_{\text {sum }}^{e}$ than the non-coordinated UAV-NOMA schemes in [9] and [10], respectively. The reason is that rather than suppressing the interference from GBS, the proposed UAV-GBS coordinated NOMA scheme takes advantage of it and makes the cell-edge users jointly served by UAV and GBS. Also, the proposed UAV-GBS coordinated NOMA outperforms the GBSs coordinated NOMA [11] by providing better air-ground channel conditions with LoS link and cost-efficient interference management with UAV's mobility.

Fig. 3 illustrates the UAV trajectories for the UAV-GBS coordinated NOMA systems with different $R_{\min }$ and $p_{t} . P_{u}=40$ mW. It can be observed from Fig. 3 that the UAV optimizes its trajectory according to $R_{\min }$ and $P_{t}$ so that the performance of cell-edge users can be maximized. When $R_{\text {min }}=2 \mathrm{bps} / \mathrm{Hz}$ and $P_{t}=0.5 \mathrm{~W}$, the trajectory of UAV is a quasi-quadrilateral connecting the four cell-edge users. As $R_{\text {min }}$ increases, the QoS

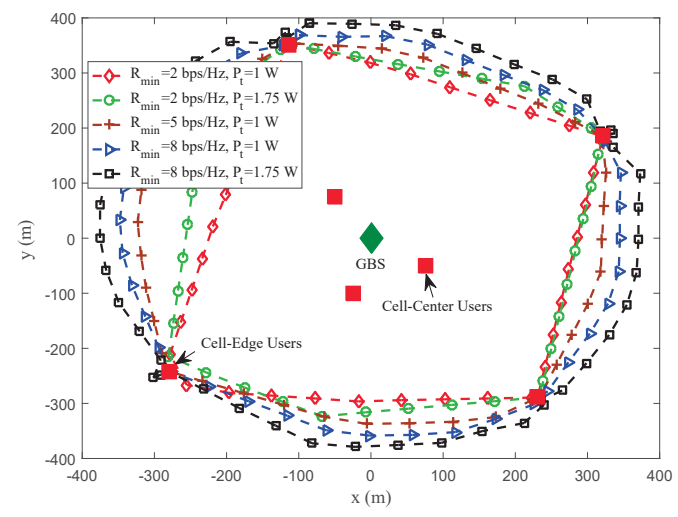

Fig. 3. UAV trajectories with different values of minimal rate requirement $R_{\min }$ and GBS transmit power $P_{t}$.

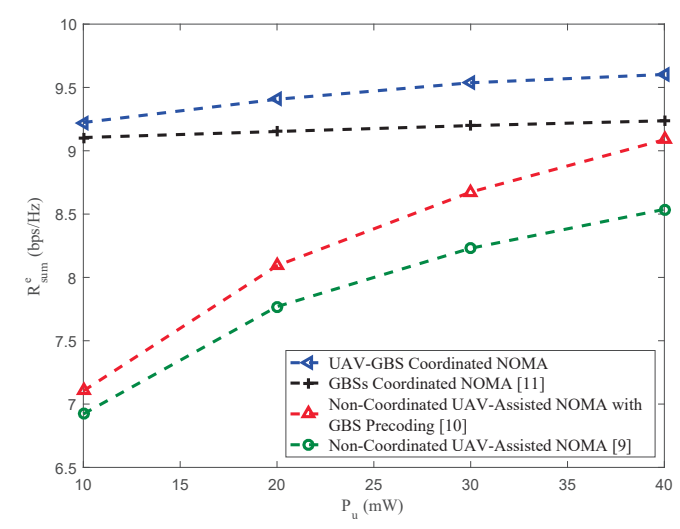

Fig. 4. Sum rate of cell-edge users with different UAV transmit power $P_{u}$.

constraint $(C 4)$ to the cell-center users becomes stricter. Also, according to (2) and $(C 3)$, with a larger $P_{t}$, the interference of cell-center users from UAV becomes the major consideration to improve $R_{\text {sum }}^{e}$, as a result, the UAV flies away from the cell-center users to reduce the interference.

Fig. 4 illustrates the performance of $R_{\text {sum }}^{e}$ for different UAVNOMA and coordinated NOMA schemes versus $P_{u}$, with $P_{t}=$ $1.5 \mathrm{~W}$ and $R_{\text {min }}=4 \mathrm{bps} / \mathrm{Hz}$. It can be learned from Fig. 4 that when $P_{u}$ is low, the proposed UAV-GBS coordinated NOMA scheme has a better performance than the non-coordinated UAV-NOMA methods [9] [10] and GBSs coordinated NOMA [11]. As $P_{u}$ becomes larger, the performance of UAV-GBS coordinated NOMA grows up more slowly than the noncoordinated UAV-NOMA with precoding [10]. That is because though the cell-edge users can be jointly served by GBS and UAV through BS coordination, the SIC interference is also introduced, which increases rapidly and has substantially effect on $R_{\text {sum }}^{e}$ when $P_{u}$ is large. It is worth noting that the design of the precoding matrix in [10] is complex and it requires additional multiple antennas to perform the precoding matrix, which cannot be directly utilized for the GBS equipped with one antenna. 
In Fig. 5, the EE performance of UAV-GBS coordinated NOMA is presented. As can be seen, the proposed UAVGBS coordinated NOMA scheme is more energy efficient at a moderate minimal rate requirement $R_{\text {min }}$. The maximum EE can be achieved at approximately $R_{\min }=9 \mathrm{bps} / \mathrm{Hz}$.

\section{CONCLUSION}

In this paper, we have proposed a UAV-GBS coordination scheme for NOMA systems to allow joint signal transmission from UAV and GBS to cell-edge users. Joint optimization of user scheduling, power allocation and UAV trajectory is conducted to maximize the sum rate of cell-edge users. A closed-form optimal solution to power allocation is derived. An SIC decoding order dedicated for UAV-GBS coordinated NOMA was proposed, based on which we presented an SOUS algorithm for both cell-center and cell-edge users. The proposed UAV-GBS coordinated NOMA scheme significantly outperforms the non-coordinated UAV-NOMA methods in [9] and [10] in terms of sum rate of cell-edge users by more than $21 \%$ and $16 \%$. It also achieves better performance than the GBSs coordinated NOMA system [11] with higher channel condition and flexibility. The proposed UAV-GBS coordinated NOMA scheme is more energy efficient at a moderate minimal rate requirement $R_{\min }$.

\section{APPENDiX: Proof OF THEOREM 1}

For a cell-center user $k \in U_{n}$, (2) can be rewritten as

$$
\sum_{j=1}^{k} P_{j, n}=\frac{\left(2^{R_{k, n}}-1\right)}{\Phi_{k, n}}+2^{R_{k, n}} \sum_{j=1}^{k-1} P_{j, n}
$$

Define $S_{k}=\sum_{j=1}^{k} P_{j, n}$ and $D_{k}=2^{\sum_{i=k+1}^{G-1} R_{i, n}}$. Multiplying $D_{k}$ at both sides of (14) yields

$$
D_{k} S_{k}=D_{k-1} S_{k-1}+\left(D_{k-1}-D_{k}\right) / \Phi_{k, n}
$$

Note that $D_{G-1}=1, S_{0}=0$. Consequently, we have

$$
S_{G-1}=\sum_{k=2}^{G-1}\left(D_{k-1}-D_{k}\right) / \Phi_{k, n}
$$

Then the sum transmit power for the cell-center users is

$\sum_{j=1}^{G-1} P_{j, n}=\frac{2^{\sum_{j=1}^{G-1} R_{j, n}}}{\Phi_{1, n}}-\frac{1}{\Phi_{G-1, n}}+\sum_{k=2}^{G-1}\left(\frac{1}{\Phi_{k-1, n}}-\frac{1}{\Phi_{k, n}}\right) 2^{\sum_{j=k+1}^{G-1} R_{j, n}}$

According to Lemma $1, \sum_{j=1}^{G-1} P_{j, n}$ is a sum of non-negative convex functions.

Then, for the cell-edge user $G$, (3) can be rewritten as

$$
\begin{aligned}
\sum_{j=1}^{G} P_{j, n} & =2^{\sum_{j=1}^{G} R_{j, n}} \frac{\sigma^{2}+V_{G, n} P_{u}}{H_{G, n}}+\sum_{k=2}^{G-1}\left(\frac{1}{\Phi_{k, n}}-\frac{1}{\Phi_{k-1, n}}\right) \\
& \times 2^{\sum_{j=k+1}^{G} R_{j, n}}+\left(\frac{\sigma^{2}}{H_{G, n}}-\frac{1}{\Phi_{G-1, n}}\right) 2^{R_{G, n}}-\frac{1}{\Phi_{G, n}}
\end{aligned}
$$

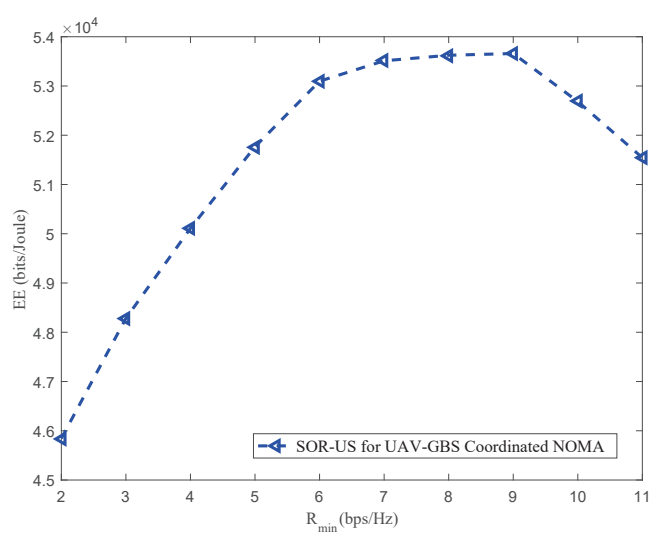

Fig. 5. EE performance for UAV-GBS coordinated NOMA systems.

Based on Lemma 1, we obtain that $\sum_{j=1}^{G-1} P_{j, n}$ is a sum of non-negative convex functions. As a result, the sum transmit power of NOMA users $\sum_{j=1}^{G} P_{j, n}$ is a convex function of $\mathbf{R}_{\mathbf{n}}$. Therefore, OP2 is equivalent to the convex problem OP3.

\section{REFERENCES}

[1] L. Dai et al., "A survey of non-orthogonal multiple access for 5G," IEEE Commun. Surv. \& Tut., vol. 20, no. 3, pp. 2294-2323, Sep. 2015.

[2] Y. Sun et al., "On the performance of network NOMA in uplink CoMP systems: a stochastic geometry approach," IEEE Trans. Commun., vol. 67, no. 7 , pp. 5084-5098, Jul. 2019.

[3] X. Pang et al., "UAV-aided NOMA networks with optimization of trajectory and precoding," in Proc. WCSP' 18, Hangzhou, China, Oct. 2018,pp. 1-6.

[4] Y. Zeng et al., Energy minimization for wireless communication with rotary-wing UAV, IEEE Trans. Wireless Commun., vol. 18, no. 4, pp. 2329-2345, Apr. 2019.

[5] X. Liu et al., "Placement and Power Allocation for NOMA-UAV Networks," IEEE Commun. Lett., pp. 1-1, Sep. 2019.

[6] Y. Liu et al., "UAV communications based on non-orthogonal multiple access" IEEE Wireless Commun., vol. 26, no. 1, pp. 52-57, Feb. 2019.

[7] J. Lyu et al., "Spectrum sharing and cyclical multiple access in UAVaided cellular offloading," in Proc. IEEE GLOBECOM'17, Singapore, Dec. 2017, pp. 1-6.

[8] F. Cheng et al., "UAV trajectory optimization for data offloading at the edge of multiple cells," IEEE Trans. Veh. Technol., vol. 67, no. 7,pp. 6732-6736, Jul. 2018.

[9] T. M. Nguyen et al., "A novel cooperative NOMA for designing UAVassisted wireless backhaul networks," IEEE J. Sel. Areas Commun., vol. 36, no. 11, pp. 2497-2507, Nov. 2018.

[10] N. Zhao et al., "Joint Trajectory and Precoding Optimization for UAVAssisted NOMA Networks," IEEE Trans. Commun., vol. 67, no. 5, pp. 3723-3735, May. 2019.

[11] A. Beylerian and T. Ohtsuki, "Coordinated non-orthogonal mulitple access (CO-NOMA)," in Proc. IEEE Globecom'16, Washington DC, USA, Dec. 2016.

[12] S. Singh et al., "Distributed approaches for inter-cell interference coordination in UAV-based LTE-Advanced hetNets," in Proc. IEEE VTCFall'18, Chicago, USA, Aug. 2018.

[13] F. Fang et al., "Energy-efficient resource allocation for downlink nonorthogonal multiple access network," IEEE Trans. Commun., vol. 64, no. 9, pp. 3722-3732, Jul. 2016.

[14] Y. Tian et al., "On the performance of opportunistic NOMA in downlink CoMP networks," IEEE Commun. Lett., vol. 20, no. 5, pp. 998-1001, Mar. 2016.

[15] Q. Wu et al., "Joint trajectory and communication design for multi-UAV enabled wireless networks," IEEE Trans. Wireless Commun., vol. 17, no. 3, pp. 2109-2121, Mar. 2018. 